\title{
Learning to be prepared
}

\author{
Willemien Kets · Mark Voorneveld
}

Accepted: 23 January 2008 / Published online: 23 February 2008

(C) The Author(s) 2008

\begin{abstract}
Behavioral economics provides several motivations for the common observation that agents appear somewhat unwilling to deviate from recent choices. More recent choices can be more salient than other choices, or more readily available in the agent's mind. Alternatively, agents may have formed habits, or use rules of thumb. This paper provides discrete-time adjustment processes for strategic games in which players display such a bias towards recent choices. In addition, players choose best replies to beliefs supported by observed play in the recent past. We characterize the limit behavior of these processes by showing that they eventually settle down in minimal prep sets (Voorneveld in Games Econ Behav 48:403-414, 2004).
\end{abstract}

Keywords Adjustment - Learning - Minimal prep sets · Availability bias · Salience · Rules of thumb

\section{JEL Classification $\quad \mathrm{C} 72 \cdot \mathrm{D} 83$}

\section{Introduction}

The behavioral economics literature provides several motivations for the common observation that agents appear somewhat unwilling to deviate from their recent choices. For instance, Tversky and Kahneman (1982, p. 11) mention the bias towards recent choices as an example of the availability bias, the ease with which instances come to mind. Similarly, Schelling (1960) has argued that players, when indifferent between

\footnotetext{
W. Kets · M. Voorneveld

Department of Econometrics and Operations Research, Tilburg University, Tilburg, The Netherlands

M. Voorneveld $(\varangle)$

Department of Economics, Stockholm School of Economics, Box 6501, 11383 Stockholm, Sweden e-mail: Mark.Voorneveld@hhs.se
} 
strategies, choose the most salient strategy. In combination with the so-called recency effect (Miller and Campbell 1959) this may explain why agents appear to have a preference for recent choices. The recency effect refers to the cognitive bias that results from disproportionate salience of recent stimuli or observations. Other motivations include models for agents displaying defaulting behavior or inertia (cf. Vega-Redondo 1993; Madrian and Shea 2001), the formation of habits (cf. Young 1998), the use of rules of thumb (cf. Ellison and Fudenberg 1993), or the locking in on certain modes of behavior due to learning by doing (cf. Grossman et al. 1977) or, as Joosten et al. (1995) express it: unlearning by not doing.

This paper provides a class of discrete-time adjustment processes for mixed extensions of finite strategic games in which players display precisely such a bias towards recent choices. Apart from this behavioral assumption, the assumptions underlying the adaptive processes in this paper are in conformance with much of the literature on learning (cf. Hurkens 1995; Fudenberg and Levine 1998; Young 1998): players choose best replies to beliefs that are supported by observed play in the recent past. The purpose of this paper is to show that these behaviorally plausible models of adaptive play eventually settle down in so-called minimal prep sets, thus providing a dynamic motivation for such sets.

Minimal prep sets ('prep' is short for 'preparation') were introduced and studied in a static framework in Voorneveld $(2004,2005)$. This set-valued solution concept for strategic games combines a standard rationality condition, stating that the set of recommended strategies to each player must contain at least one best reply to whatever belief he may have that is consistent with the recommendations to the other players, with players' aim at simplicity, which encourages them to maintain a set of strategies that is as small as possible. The latter feature discerns minimal prep sets from (a) minimal curb sets (Basu and Weibull 1991), which are product sets of pure strategies containing not just some, but all best responses against beliefs restricted to the recommendations to the remaining players, and (b) persistent retracts (Kalai and Samet 1984), which also require the recommendations to each player to contain at least one best reply to beliefs in a small neighborhood of the beliefs restricted to the recommendations to the other players.

The choice of the term "preparation" in connection with minimal prep sets is motivated by the rationality requirement. Given an arbitrary belief of a player that is consistent with the recommendations to the other players, his recommended set of strategies leaves him well-prepared: it contains an optimal response against all such eventualities. On the other hand, one does not have to be exhaustive to be prepared: the notion of prep sets avoids the potential snowball effect from the requirement that all best replies against a given belief (and all best replies against all these best replies, and so on...) need to be included, as demanded of the curb sets of Basu and Weibull (1991). Think of the set of recommendations to a player in a minimal prep set as a well-packed suitcase for a holiday: you want to be prepared for different kinds of weather, but bringing all five of your umbrellas and all seven bathing suits may be overdoing it. ${ }^{1}$

\footnotetext{
1 We are grateful to Dries Vermeulen for suggesting this "no excess luggage" interpretation.
} 
Fig. 1 A $3 \times 3$ game

\begin{tabular}{c|c|c|c|}
\multicolumn{1}{c}{} & \multicolumn{1}{c}{$C_{1}$} & \multicolumn{1}{c}{$C_{2}$} & $C_{3}$ \\
\cline { 2 - 4 }$R_{1}$ & $1,-1$ & $-1,1$ & $-100,-100$ \\
\cline { 2 - 4 }$R_{2}$ & $-1,1$ & $1,-1$ & $-1,1$ \\
\cline { 2 - 4 }$R_{3}$ & $-100,-100$ & $-1,1$ & $1,-1$ \\
\cline { 2 - 4 } & &
\end{tabular}

The game in Fig. 1 provides a simple example to illustrate the difference between pure Nash equilibria, minimal curb sets, and minimal prep sets. The game has no pure Nash equilibria. Its only-hence minimal — curb set is the entire pure strategy space $\left\{R_{1}, R_{2}, R_{3}\right\} \times\left\{C_{1}, C_{2}, C_{3}\right\}$. There are two minimal prep sets, $\left\{R_{1}, R_{2}\right\} \times\left\{C_{1}, C_{2}\right\}$ and $\left\{R_{2}, R_{3}\right\} \times\left\{C_{2}, C_{3}\right\}$, roughly speaking the "Matching pennies" subgames.

Voorneveld $(2004,2005)$ contains a general existence proof and a detailed comparison of minimal prep sets with Nash equilibria, rationalizability, minimal curb sets, and persistent retracts. Voorneveld et al. (2005) provide axiomatizations of minimal prep sets and minimal curb sets. Tercieux and Voorneveld (2005) show that minimal prep sets provide sharp predictions in many economic applications, including potential games, congestion games, and supermodular games, even in cases where minimal curb sets have no cutting power whatsoever and simply consist of the entire strategy space. The current paper complements this literature by providing a dynamic motivation for minimal prep sets.

For play to settle down in a specific set, like a minimal prep or curb set, players somehow need to learn to coordinate on actions from within this set. Crawford and Haller (1990, p. 577) indicate that an important coordination device is the fact that players "use asymmetric history to "label" actions that cannot be distinguished at the start". In our model, players use histories to form beliefs, but also to select between best replies. The behavioral bias towards recent best replies is a specific example of a way to exploit the history to distinguish between actions that all perform well against a player's beliefs.

The work that is closest in spirit to our analysis is that of Hurkens (1995). In both his work and in the current paper, convergence to a set-valued solution concept is established, firstly, for discrete-time adjustment processes characterized by conditions on transition probabilities (zero or positive), secondly, for all finite games (in contrast with e.g., Young (1998), who restricts attention to weakly acyclic games), and, thirdly, for all memory lengths exceeding a certain lower bound. There are, however, important differences. The behavioral bias towards recent choices that players use to distinguish between best replies is absent in Hurkens' model: there, players indiscriminately choose best replies to their beliefs. As a consequence, players in our model need to keep track of whether one best reply was chosen more recently than another. However, this does not mean that a player needs to have perfect memory of his own past action choices. This is particularly clear if a player has only two actions: if both happen to be a best reply to his current belief, the action he chose in the previous round is the most recent one and therefore all he needs to recall. We return to this issue in more detail in Remarks 3.1 and 5.2.

The outline of this paper is as follows. We recall definitions in Sect. 2. The evolution of play is discussed in Sect. 3. Section 4 contains the convergence theorem and explains 
the steps towards the proof. In Sect. 5, we discuss a more general class of adjustment processes for which play also settles down in minimal prep sets. Section 6 contains concluding remarks. All proofs are contained in the appendix.

\section{Preliminaries}

Weak set inclusion is denoted by $\subseteq$, strict set inclusion by $\subset$. The number of elements in a finite set $S$ is denoted by $|S|$. For $k \in \mathbb{N}$, the $k$-fold cartesian product $\times_{i=1}^{k} S$ is denoted by $S^{k}$.

A game is a tuple $G=\left\langle N,\left(A_{i}\right)_{i \in N},\left(u_{i}\right)_{i \in N}\right\rangle$, where $N=\{1, \ldots, n\}$ is a nonempty, finite set of players, each player $i \in N$ has a nonempty, finite set $A_{i}$ of pure strategies/actions and a von Neumann-Morgenstern utility function $u_{i}: A \rightarrow \mathbb{R}$ on the set of pure strategy profiles $A=\times_{i \in N} A_{i}$. Let $X_{i}$ be a nonempty subset of $A_{i}$. The set of mixed strategies of player $i \in N$ with support in $X_{i}$ is denoted by $\Delta\left(X_{i}\right)$. Payoffs are extended to mixed strategies in the usual way. Let $i \in N$ and let $\alpha_{-i} \in \times_{j \in N \backslash\{i\}} \Delta\left(A_{j}\right)$ be a belief ${ }^{2}$ of player $i$. The set

$$
B R_{i}\left(\alpha_{-i}\right)=\left\{a_{i} \in A_{i} \mid \forall b_{i} \in A_{i}: u_{i}\left(a_{i}, \alpha_{-i}\right) \geq u_{i}\left(b_{i}, \alpha_{-i}\right)\right\}
$$

is the set of pure best responses of player $i$ against $\alpha_{-i}$.

Fix a game $G=\left\langle N,\left(A_{i}\right)_{i \in N},\left(u_{i}\right)_{i \in N}\right\rangle$. A prep set (Voorneveld 2004) is a nonempty product set $X=\times_{i \in N} X_{i} \subseteq A$ of pure-strategy profiles such that for each $i \in N$ and each belief $\alpha_{-i}$ of player $i$ with support in $X_{-i}$, the set $X_{i}$ contains at least one best response of player $i$ against his belief:

$$
\forall i \in N, \forall \alpha_{-i} \in \times_{j \in N \backslash\{i\}} \Delta\left(X_{j}\right): \quad B R_{i}\left(\alpha_{-i}\right) \cap X_{i} \neq \emptyset .
$$

A prep set $X$ is minimal if no prep set is a proper subset of $X$. Establishing existence of minimal prep sets in finite games is simple: the entire pure-strategy space $A$ is a prep set. Hence the collection of prep sets is nonempty, finite (since $A$ is finite) and partially ordered by set inclusion. Consequently, a minimal prep set exists. See Voorneveld (2004, Theorem 3.2) for a general existence result.

In our adaptive processes, a game $G=\left\langle N,\left(A_{i}\right)_{i \in N},\left(u_{i}\right)_{i \in N}\right\rangle$ is played once every period in discrete time. A history (of play) is a sequence $h=\left(a^{1}, \ldots, a^{L}\right) \in A^{L}$ of some arbitrary length $L \in \mathbb{N}$, whose leftmost element

$$
\ell(h):=a^{1} \in A
$$

is interpreted as the action profile chosen in the previous period according to history $h$, with $\ell_{i}(h):=a_{i}^{1} \in A_{i}$ the action played by $i \in N$. Generally, the $k$-th element from the left is the action profile $a^{k} \in A$ chosen $k \in \mathbb{N}$ periods ago.

\footnotetext{
2 Beliefs are profiles of mixed strategies: correlation is not allowed.
} 
A successor of history $h=\left(a^{1}, \ldots, a^{L}\right)$ is a history obtained after one more period of play, a history $h^{\prime}=\left(b^{1}, b^{2}, \ldots, b^{L+1}\right)$ obtained from $h$ by appending a new leftmost element: $b^{1} \in A$ and $b^{k}=a^{k-1}$ for all $k=2, \ldots, L+1$.

Fix a history $h=\left(a^{1}, \ldots, a^{L}\right)$ and a player $i \in N$. The set of actions chosen by $i$ during the last ${ }^{3} k \in\{1, \ldots, L\}$ rounds of history $h$ is denoted by

$$
\lambda_{i}(h, k):=\left\{a_{i}^{1}, \ldots, a_{i}^{k}\right\} .
$$

The order $o_{i, h}$ of player $i$ 's actions in history $h$ is defined as follows: his most recent action, i.e., the first encountered action is $o_{i, h}(1):=a_{i}^{1}$ and, inductively, for $k=$ $2, \ldots,\left|\left\{a_{i}^{1}, \ldots, a_{i}^{L}\right\}\right|$, the $k$-th encountered action is $o_{i, h}(k):=a_{i}^{m}$ with $m=\min \{q \in$ $\left.\{1, \ldots, L\} \mid a_{i}^{q} \notin\left\{o_{i, h}(1), \ldots, o_{i, h}(k-1)\right\}\right\}$.

Example 2.1 Consider a two-player game with $N=\{1,2\}$ and action spaces $A_{1}=$ $\left\{R_{1}, R_{2}\right\}, A_{2}=\left\{C_{1}, C_{2}\right\}$. Consider the history

$$
h=\left(\left(R_{1}, C_{2}\right),\left(R_{2}, C_{2}\right),\left(R_{2}, C_{1}\right)\right)
$$

of length three. Then $\ell(h)=\left(R_{1}, C_{2}\right)$. The set of actions player 1 chose during the most recent two periods is $\lambda_{1}(h, 2)=\left\{R_{1}, R_{2}\right\}$, whereas $\lambda_{2}(h, 2)=\left\{C_{2}\right\}$. As to orders, player 1's action $R_{1}$ is encountered first, then $R_{2}$, so $o_{1, h}(1)=R_{1}, o_{1, h}(2)=R_{2}$. Similarly, $o_{2, h}(1)=C_{2}, o_{2, h}(2)=C_{1}$.

\section{Adaptive play}

This section presents a class of Markov chains to model adaptive play with a bias towards choices from the recent past. A game $G=\left\langle N,\left(A_{i}\right)_{i \in N},\left(u_{i}\right)_{i \in N}\right\rangle$ is played once every period in discrete time. In line with much of the literature on learning models (cf. Hurkens 1995; Fudenberg and Levine 1998; Young 1998), players choose, at each moment in time, best replies to beliefs supported by a limited horizon of observed past play of fixed length $T \in \mathbb{N} .{ }^{4}$ Consequently, we define the state space $H$ to consist of all histories $h=\left(a^{1}, \ldots, a^{L}\right)$ with length at least $T$, i.e., $h \in \cup_{K \in \mathbb{N}, K \geq T} A^{K}$.

Having defined the set $H$ of states, we proceed to transition probability functions $P: H \times H \rightarrow[0,1]$, where $P\left(h, h^{\prime}\right)$ is the probability of moving from state $h \in H$ to state $h^{\prime} \in H$ in one period and $\sum_{h^{\prime} \in H} P\left(h, h^{\prime}\right)=1$ for all $h \in H$. To do so, we model beliefs and responses to them.

Beliefs: Players' beliefs are based on observed play in the past $T \in \mathbb{N}$ periods. Formally, for each state $h \in H$, if the sequence of action profiles played in the past $T$ periods is $\left(a^{1}, \ldots, a^{T}\right) \in A^{T}$, then player $i$ 's beliefs are drawn from a probability measure $\mathbb{P}_{\left(i,\left(a^{1}, \ldots, a^{T}\right)\right)}$ over the set of beliefs (with its standard topology and Borel $\sigma$-algebra)

\footnotetext{
3 Hence our choice of the alliterative $\lambda$ (lambda).

4 Our adjustment processes are defined for a fixed game $G$ and memory length $T$; to simplify notation, indices $G$ and $T$ are suppressed.
} 


$$
\times_{j \in N \backslash\{i\}} \Delta\left(\left\{a_{j}^{1}, \ldots, a_{j}^{T}\right\}\right)=\times_{j \in N \backslash\{i\}} \Delta\left(\lambda_{j}(h, T)\right)
$$

with support in the product set of actions chosen in the previous $T$ periods. For the convergence result, the exact probabilities are irrelevant: what matters is that some are positive, others zero. We therefore refrain from restricting attention to specific belief formation processes or updating procedures. As long as beliefs are sufficiently diverse — see Remark 3.2 or the related discussion in Hurkens (1995, pp. 310-311) — it is immaterial how they are formed.

Responses: Given a belief $\alpha_{-i} \in \times_{j \in N \backslash\{i\}} \Delta\left(\lambda_{j}(h, T)\right)$, we assume that player $i$ chooses the most recent best reply to $\alpha_{-i}$ if such a best reply exists, i.e., if in state $h$ some best reply to $\alpha_{-i}$ has been played before. Otherwise, player $i$ chooses each best reply to $\alpha_{-i}$ with positive probability, i.e., it is drawn from a probability measure $\mathbb{P}_{\alpha_{-i}}$ over $A_{i}$ whose support coincides with the set of best replies $B R_{i}\left(\alpha_{-i}\right)$. Players thus have a bias towards recent choices. ${ }^{5}$

Together, the probability distributions $\mathbb{P}_{\left(i,\left(a^{1}, \ldots, a^{T}\right)\right)}$ that fix for each player $i \in N$ and account of recent play $\left(a^{1}, \ldots, a^{T}\right) \in A^{T}$ the way beliefs are drawn, and the assumption that players are biased towards recent choices, determine the transition probabilities $P\left(h, h^{\prime}\right) \in[0,1]$ for each pair of states $\left(h, h^{\prime}\right) \in H \times H$. If $P\left(h, h^{\prime}\right)>0$, then histories $h, h^{\prime} \in H$ satisfy conditions P1 and P2 in Fig. 2.

Condition P1 is standard for discrete-time processes, stating that between time periods the game is played once: the process moves from a history $h$ to one of its successors $h^{\prime}$. Condition $\mathrm{P} 2$ states, firstly, that the process $P$ is a best-reply process: the action $\ell_{i}\left(h^{\prime}\right) \in A_{i}$ chosen by each player $i \in N$ is a best reply to some belief $\alpha_{-i}$ about the remaining players' behavior based on recent experience, i.e., with support in $\times_{j \in N \backslash\{i\}} \Delta\left(\lambda_{j}(h, T)\right)$. Secondly, it models the bias towards recent choices: whenever possible, each player $i \in N$ chooses the most recent best reply to belief $\alpha_{-i}$.

Let $\mathscr{P}$ be the class of transition probability functions $P$ achieved in this way, i.e., using probability distributions $\left\{\mathbb{P}_{\left(i,\left(a^{1}, \ldots, a^{T}\right)\right)} \mid i \in N,\left(a^{1}, \ldots, a^{T}\right) \in A^{T}\right\}$ and the behavioral bias, and with $P\left(h, h^{\prime}\right)>0$ if and only if states $h, h^{\prime} \in H$ satisfy conditions $\mathrm{P} 1$ and $\mathrm{P} 2$ in Fig. 2.

Remark 3.1 The behavioral bias towards recent choices modeled in $\mathrm{P} 2$ requires that a player with multiple best replies against his current belief recalls whether one of them was played more recently than another. However, this does not require players to have perfect memory about their own actions: If you played one best reply yesterday and another a week ago, your choice is independent of whether you also adopted these actions further away in the past. All that matters is that each player $i \in N$ in history $h \in H$ recalls the order $o_{i, h}$ defined in Sect. 2. This is a considerably more modest requirement than remembering the entire history of own actions: $o_{i, h}$ specifies a simple

$\overline{5 \text { The probability of choosing } a_{i}} \in A_{i}$ against beliefs to which no best reply was chosen before is

$$
\int_{\left\{\alpha_{-i} \mid B R_{i}\left(\alpha_{-i}\right) \cap \lambda_{i}(h, L)=\emptyset\right\}} \mathbb{P}_{\alpha_{-i}\left(a_{i}\right) \mathrm{d} \mathbb{P}_{\left(i,\left(a^{1}, \ldots, a^{T}\right)\right)},}
$$

i.e., $\alpha_{-i} \mapsto \mathbb{P}_{\alpha_{-i}}\left(a_{i}\right)$ is assumed to be Borel measurable. 


\begin{tabular}{|l|l|}
\hline P1 & $h^{\prime}$ is a successor of $h:=\left(a^{1}, \ldots, a^{L}\right)$. \\
\hline P2 & For each $i \in N, \ell_{i}\left(h^{\prime}\right)$ is a best reply to some belief $\alpha_{-i} \in \times_{j \in N \backslash\{i\}} \Delta\left(\lambda_{j}(h, T)\right)$. \\
& It is the most recent best reply, if such a best reply exists. Formally: \\
& $\bullet$ \\
& $\ell_{i}\left(h^{\prime}\right) \in B R_{i}\left(\alpha_{-i}\right)$ \\
& $\bullet$ \\
& if $B R_{i}\left(\alpha_{-i}\right) \cap\left\{a_{i}^{1}, \ldots, a_{i}^{L}\right\} \neq \emptyset$, then $\ell_{i}\left(h^{\prime}\right)=a_{i}^{k}$, where \\
& $k=\min \left\{m \in\{1, \ldots, L\} \mid a_{i}^{m} \in B R_{i}\left(\alpha_{-i}\right)\right\}$.
\end{tabular}

Fig. 2 For $P \in \mathscr{P}, P\left(h, h^{\prime}\right)>0$ iff $h, h^{\prime} \in H$ satisfy $\mathrm{P} 1$ and $\mathrm{P} 2$

linear order of at most $\left|A_{i}\right|$ actions. Between consecutive rounds of play, this linear order either remains the same or changes in the following way: The action ranked first (the most recent action) is changed and the other actions are moved one step down the ladder. For instance, even after numerous rounds of play, the only thing a player with just two actions needs to recall from his own past is last period's action.

Remark 3.2 Inherent in the definition of the class $\mathscr{P}$ of transition probability functions (see Fig. 2) is that beliefs must be "sufficiently diverse" to assure that player $i \in N$ has a positive probability of selecting $a_{i} \in A_{i}$ whenever it is a (most recent) best reply to some belief over recent past play. More specifically, by $\mathrm{P} 2$, player $i$ is tempted to play $a_{i}$ against beliefs $\alpha_{-i}$ over recent past play to which it is the most recent best reply or-if no such most recent best reply exists - to which it is an arbitrary best reply. If the set of such "tempting" beliefs is nonempty, player $i$ assigns positive probability to it.

For each $k \in \mathbb{N}, P^{k}: H \times H \rightarrow[0,1]$ denotes the $k$-step transition probabilities of the Markov process with transition probability function $P \in \mathscr{P}: P^{1}=P$ and $P^{k}=P \circ P^{k-1}$ if $k>1$.

\section{Convergence and steps towards the proof}

This section presents the main result of this paper. Theorem 4.1 states, for each game $G$ and adjustment process in the class $\mathscr{P}$, that if beliefs are based on recent experience of sufficient length $T$, then play will eventually settle down within a minimal prep set. The steps of the proof are briefly explained in this section; the proof itself is contained in Appendix A.

Given a game $G$ and an adjustment process $P \in \mathscr{P}$, we say that the process eventually settles down in a minimal prep set of $G$ if the probability that the process after $k$ steps is in a state $h \in H$ where

- the most recently played action profile $\ell(h)$ lies in some minimal prep set $X$ of $G$ :

$$
\ell(h) \in X
$$

- all future action profiles remain inside $X$ :

$$
\ell\left(h^{\prime}\right) \in X \text { whenever } P^{m}\left(h, h^{\prime}\right)>0 \text { for some } m \in \mathbb{N}, h^{\prime} \in H,
$$

converges to one as $k$ goes to infinity. 
Theorem 4.1 Let $G=\left\langle N,\left(A_{i}\right)_{i \in N},\left(u_{i}\right)_{i \in N}\right\rangle$ be a game. Let the horizon $T \in \mathbb{N}$ of recent past play on which beliefs are based satisfy

$$
T \geq \max \left\{\sum_{i \in N}\left|A_{i}\right|-n+1,2\left|A_{1}\right|, \ldots, 2\left|A_{n}\right|\right\} .
$$

If $P \in \mathscr{P}$, then play eventually settles down in a minimal prep set of $G$.

Remark (i) If the game has several minimal prep sets, the one selected by the learning process typically depends on initial conditions. For instance, if the initial state is such that the collection of most recent actions is a minimal prep set $X$, the process settles down in $X$.

(ii) Condition P2 assures that play will not settle down in proper subsets of a minimal prep set. Suppose play settles down in a product set $Y$ properly contained in a minimal prep set $X$. Since $Y$ is not a prep set, there is a player $i$ with a belief over recent past play against which $Y_{i}$ contains no best reply. Condition P2 assures that player $i$ with positive probability chooses such a best reply, i.e., an action outside $Y_{i}$, contradicting the assumption that play has settled down in $Y$. A similar intuition is used in the proof of the Theorem in Appendix A (Lemma A.1).

(iii) The statement of Theorem 4.1 follows the traditional pattern (cf. Hurkens 1995; Fudenberg and Levine 1998; Young 1998): if memory is 'sufficiently long', play settles down in sets of a certain type. Thus, we indicate a sufficient length in (1), without aiming at sharpness. Were one to exploit specific features of a game, the bound might be decreased (e.g., Kets and Voorneveld 2007).

Steps of the proof The proof of Theorem 4.1 proceeds in four steps:

Step 1 Let $h_{0} \in H$. The process moves with positive probability in $T-1$ steps to a state $h_{1} \in H$ where the product set $\times_{i \in N} \lambda_{i}\left(h_{1}, T\right) \subseteq A$ of actions played in the past $T$ periods is a prep set.

The intuition behind this step is as follows. If, for some state $g \in H$ and some $k \leq T$, the product set $\times_{i \in N} \lambda_{i}(g, k)$ is a prep set, then with positive probability, players choose actions from this prep set for $T-k$ periods in a row. If on the other hand, $\times_{i \in N} \lambda_{i}(g, k)$ is not a prep set, then there is a nonempty set of players $i \in N$ with a belief $\alpha_{-i}^{*} \in \times_{j \in N \backslash\{i\}} \Delta\left(\lambda_{j}(g, k)\right)$ over play in the past $k$ periods to which $\lambda_{i}(g, k)$ does not contain a best reply. In that case, one can construct a sequence of states $g_{1}, g_{2}, \ldots \in H$ with $g_{1}=g, P\left(g_{k}, g_{k+1}\right)>0$ for all $k=1,2, \ldots$, such that the sequence of product sets $\times_{i \in N} \lambda_{i}\left(g_{k}, k\right)$ is strictly increasing with respect to set inclusion (see Lemma A.1 in Appendix A). All these sets are contained in the finite set $A$ of action profiles which is a prep set. Since there are only finitely many actions, the sequence reaches, after a finite number of steps, a state $g_{K} \in H$ where $\times_{i \in N} \lambda_{i}\left(g_{K}, K\right)$ is a prep set. From that state onwards, players choose with positive probability actions from the prep set for $T-K$ periods in a row.

Step 2 From state $h_{1}$, the process moves with positive probability in a finite number of steps to a state $h_{2} \in H$ where $X:=\times_{i \in N} \lambda_{i}\left(h_{2}, T\right)$ is a minimal prep set. 
Indeed, let $X=\times_{i \in N} X_{i} \subseteq \times_{i \in N} \lambda_{i}\left(h_{1}, T\right)$ be a minimal prep set. The proof of this step relies on the fact that one can-under some conditions-perform so-called neighbor switches: from a state $h \in H$, the process moves with positive probability in $T$ steps to a state $h^{\prime} \in H$ whose horizon of recent past play is identical to the one in $h$, except that two neighboring actions of some player have changed places (see Lemma A.6). As all permutations of a finite set can be obtained by a chain of such neighbor switches, the process moves with positive probability from state $h_{1}$ to a state $h^{\prime}$ where, for each player $i \in N, \lambda_{i}\left(h^{\prime},\left|X_{i}\right|\right)=X_{i}$, i.e., the $\left|X_{i}\right|$ most recent actions of each player $i$ are exactly those in his component of the minimal prep set $X$. Then it is easy to show that the process moves with positive probability to a state $h_{2}$ within a finite number of steps such that $\times_{i \in N} \lambda_{i}\left(h_{2}, T\right)=X$ is a minimal prep set.

Step 3 After reaching state $h_{2}$, all action profiles that are played with positive probability lie in $X$, i.e.,

$$
\forall k \in \mathbb{N}, \quad \forall h \in H: P^{k}\left(h_{2}, h\right)>0 \Rightarrow \ell(h) \in X .
$$

In state $h_{2}, \times_{i \in N} \lambda_{i}\left(h_{2}, T\right)=X$ is a minimal prep set, which by definition contains at least one best reply to whatever belief a player may have about other players' choices from $X$. Hence, by induction, the actions from minimal prep set $X$ will always be fresher in players' recollection of past play than actions outside $X$, so that to any belief that a player $i$ may have about opponents' play, there is an action in $X_{i}$ that is the most recent best reply. Hence, from state $h_{2}$ onwards, players $i \in N$ only choose actions from $X_{i}$.

Step 4 Starting from an arbitrary history $h_{0}$, Steps 1 and 2 show that there is a positive probability of proceeding to a history $h_{2}$ in a finite number of steps, after which play settles down in a minimal prep set, i.e., a positive probability of proceeding to an absorbing set of states in finitely many steps. Since the initial history was chosen arbitrarily, this eventually happens with probability one, finishing the proof.

\section{Allowing for other behavioral biases}

To show that processes from $\mathscr{P}$ eventually settle down in minimal prep sets, the proof of Steps 1 and 2 of Theorem 4.1 (see Appendix A) uses that certain transition probabilities are positive to show that the process can move from any initial state $h_{0} \in H$ in a finite number of steps to a state $h_{2} \in H$ where $\times_{i \in N} \lambda_{i}\left(h_{2}, T\right)$ is a minimal prep set. The proof of Step 3 uses that certain transition probabilities are zero to show that each player-once such a state $h_{2}$ is reached-continues to play action profiles from the minimal prep set. We motivated these conditions on the transition probabilities by assuming that players, whenever possible, choose the most recent best reply to a certain belief. However, any class of adjustment processes that respects these conditions on the sign of the transition probabilities will converge to minimal prep sets. Hence, one can easily extend the class of adjustment processes with this limit behavior. 
In particular, suppose that for each player $i \in N$, the response to a belief drawn from recent past play is chosen according to a probability distribution (mixed strategy) $R_{i, h} \in \Delta\left(A_{i}\right)$ depending on (1) the account $\left(a^{1}, \ldots, a^{T}\right)$ of recent past play, and (2) the order in which the players' used actions appear in $h$. That is, for each pair of states $h=\left(a^{1}, \ldots, a^{L}\right), g=\left(b^{1}, \ldots, b^{K}\right) \in H$ :

$$
\left.\begin{array}{c}
\left(a^{1}, \ldots, a^{T}\right)=\left(b^{1}, \ldots, b^{T}\right) \\
o_{i, h}=o_{i, g} \text { for all } i \in N
\end{array}\right\} \Rightarrow R_{i, h}=R_{i, g} \text { for all } i \in N .
$$

The collection of functions $R=\left(R_{i, h}\right)_{i \in N, h \in H}$ determines, for each pair of states $h, h^{\prime} \in H$, the transition probability $P_{R}\left(h, h^{\prime}\right) \in[0,1]$. If $P_{R}\left(h, h^{\prime}\right)>0$, then $h^{\prime}$ is a successor of $h$ (property P1 in Fig. 2) and

$$
P_{R}\left(h, h^{\prime}\right)=\prod_{i \in N} R_{i, h}\left(\ell_{i}\left(h^{\prime}\right)\right)
$$

is the probability of the players choosing action profile $\ell\left(h^{\prime}\right)$. Let $\widetilde{\mathscr{P}}$ be the collection of transition probability functions $\left\{P_{R}: H \times H \rightarrow[0,1] \mid R=\left(R_{i, h}\right)_{i \in N, h \in H}\right\}$ satisfying the restrictions on the sign of the transition probabilities instrumental to the proof of Theorem 4.1, i.e., for each pair of histories $h, h^{\prime} \in H$ :

$(\alpha)$ If P1 and P2 hold, then $P_{R}\left(h, h^{\prime}\right)>0$.

( $\beta$ ) If the product set of actions played during the most recent $k \geq T$ rounds of $h$ is a minimal prep set, play settles down within this set. Formally, if $X:=$ $\times_{i \in N} \lambda_{i}(h, k)$ is a minimal prep set for some $k \geq T$ and $P_{R}\left(h, h^{\prime}\right)>0$, then $\times_{i \in N} \lambda_{i}\left(h^{\prime}, k+1\right)=X$, i.e., $\ell\left(h^{\prime}\right) \in X$.

One easily shows that $\mathscr{P} \subseteq \widetilde{\mathscr{P}}$. In many games, the set inclusion is strict and one finds processes in $\widetilde{\mathscr{P}} \backslash \mathscr{P}$ by letting players choose more freely among recent best replies. By construction, if memory is sufficiently long, processes in $\widetilde{\mathscr{P}}$ eventually settle down in minimal prep sets. To summarize (the proof is in Appendix B):

Proposition 5.1 Let $G=\left\langle N,\left(A_{i}\right)_{i \in N},\left(u_{i}\right)_{i \in N}\right\rangle$ be a game andlet $T \in \mathbb{N}$. Then $\mathscr{P} \subseteq \widetilde{\mathscr{P}}$. Moreover, if $P_{R} \in \widetilde{\mathscr{P}}$ and the horizon $T \in \mathbb{N}$ of recent past play is sufficiently large, then play eventually settles down in a minimal prep set of $G$.

Finally, we show why it is essential for convergence to minimal prep sets that players keep track of the order $o_{i, h}$ defined in Sect. 2, rather than just the order of their actions over the past $T$ rounds of play.

Remark 5.2 If players remember from their own past only their actions in the previous $T$ periods, the resulting processes need not converge to minimal prep sets: None of the players $i \in N$ can condition his behavior on the order $o_{i, h}$ of actions chosen more than $T$ periods ago at state $h$. To see why this prevents convergence to minimal prep sets, refer back to Fig. 1. Suppose that over the past $T$ rounds, players have chosen the actions from minimal prep set $X=\left\{R_{1}, R_{2}\right\} \times\left\{C_{1}, C_{2}\right\}$. Why wouldn't play settle down there? Suppose players play $\left(R_{1}, C_{2}\right)$ at a given round, which are best replies to beliefs over $X$. In response to these actions, there is a positive probability 
that they choose $\left(R_{2}, C_{2}\right)$ for $T$ consecutive periods. At that point, player 2's only feasible belief over past play is that player 1 chooses $R_{2}$. Player 2 recalls only his past $T$ actions, i.e., just $C_{2}$ which is no best reply to $R_{2}$. Therefore, he chooses among the best replies $\left\{C_{1}, C_{3}\right\}$ to $R_{2}$, which means that he may jump outside the minimal prep set $X$ by selecting $C_{3}$.

\section{Concluding remarks}

The purpose of this paper was to study discrete-time best-response processes with a behaviorally plausible bias towards recent actions. Such processes were shown to settle down in minimal prep sets. This dynamic motivation complements earlier papers on minimal prep sets in a static environment, where the concept is compared with many other solution concepts (Voorneveld 2004, 2005; Voorneveld et al. 2005) and shown to have genuine "bite" in economic applications (Tercieux and Voorneveld 2005), even in cases where, for instance, minimal curb sets have no cutting power whatsoever.

Several modifications of these processes were discussed in the previous section. We cannot possibly do justice to the long list of choice biases discussed in the behavioral economics literature. An interesting direction for future research-although outside the scope of the current paper-would be to more systematically investigate the links between different types of behaviorally plausible biases in adjustment processes and the corresponding limiting behavior.

Acknowledgments Financial support from the Netherlands Organization for Scientific Research (NWO) and the Wallander/Hedelius Foundation is gratefully acknowledged. We thank Kaushik Basu, Wieland Müller, Hans Peters, Dolf Talman, Dries Vermeulen, Jörgen Weibull, and several audiences for helpful comments and discussions. We thank two referees and an associate editor of this journal for their careful reading and very concrete suggestions for improvements.

Open Access This article is distributed under the terms of the Creative Commons Attribution Noncommercial License which permits any noncommercial use, distribution, and reproduction in any medium, provided the original author(s) and source are credited.

\section{Appendix A Proof of Theorem 4.1}

Fix a game $G=\left\langle N,\left(A_{i}\right)_{i \in N},\left(u_{i}\right)_{i \in N}\right\rangle$, length $T \in \mathbb{N}$ of recent past play with $T \geq \max \left\{\sum_{i \in N}\left|A_{i}\right|-n+1,2\left|A_{1}\right|, \ldots, 2\left|A_{n}\right|\right\}$, and an adjustment process with transition probability function $P \in \mathscr{P}$. We start with some additional notation. Fix an arbitrary history $h=\left(a^{1}, \ldots, a^{L}\right) \in H$ and player $i \in N$. The action player $i$ chose in $h$ a number of $t \in\{1, \ldots, T\}$ periods ago is denoted by

$$
a_{i}(h, t):=a_{i}^{t}
$$

and the action player $i$ chose in $h$ exactly $T$ periods ago is denoted by

$$
\tau_{i}(h):=a_{i}^{T}=a_{i}(h, T)
$$


Action $a_{i} \in \lambda_{i}(h, T)$ is blocked in $h$ if there is no belief $\alpha_{-i} \in \times_{j \in N \backslash\{i\}} \Delta\left(\lambda_{j}(h, T)\right)$ against which it is the most recent best reply. Finally, the frequency with which player $i$ chose action $a_{i} \in \lambda_{i}(h, T)$ during the past $T$ rounds of history $h$ is

$$
f_{i}\left(h, a_{i}\right)=\left|\left\{t \in\{1, \ldots, T\}: a_{i}(h, t)=a_{i}\right\}\right| .
$$

We now prove the four steps of Theorem 4.1.

\section{A.1 Proof of Step 1}

Step 1 Let $h_{0} \in H$. The process moves with positive probability in $T-1$ steps to a state $h_{1} \in H$ where the product set $\times_{i \in N} \lambda_{i}\left(h_{1}, T\right) \subseteq A$ of actions played in the past $T$ periods is a prep set. The proof uses the following lemma.

Lemma A.1 Consider state $h=\left(a^{1}, \ldots, a^{L}\right) \in H$ and a number $t \in\{1, \ldots, T-1\}$.

(a) Suppose that $\times_{i \in N} \lambda_{i}(h, t) \subseteq A$ is not a prep set. Then the process moves with positive probability to a successor $h^{\prime}$ of $h$ where

$$
\times_{i \in N} \lambda_{i}(h, t) \subset \times_{i \in N} \lambda_{i}\left(h^{\prime}, t+1\right)
$$

(b) Suppose that $\times_{i \in N} \lambda_{i}(h, t) \subseteq A$ is a prep set. Then the process moves with positive probability to a successor $h^{\prime}$ of $h$ where

$$
\times_{i \in N} \lambda_{i}(h, t)=\times_{i \in N} \lambda_{i}\left(h^{\prime}, t+1\right) .
$$

Proof (a) Since $\times_{i \in N} \lambda_{i}(h, t) \subseteq A$ is not a prep set, there is a nonempty set $S \subseteq N$ of players $i \in N$ with a belief $\alpha_{-i}^{*} \in \times_{j \in N \backslash\{i\}} \Delta\left(\lambda_{j}(h, t)\right)$ over the play in the past $t$ periods to which $\lambda_{i}(h, t)$ does not contain a best reply: $B R_{i}\left(\alpha_{-i}^{*}\right) \cap$ $\lambda_{i}(h, t)=\emptyset$. Fix such a belief $\alpha_{-i}^{*}$ for each $i \in S$ and let $b_{i} \in B R_{i}\left(\alpha_{-i}^{*}\right)$ be a best reply to $\alpha_{-i}^{*}$ chosen in accordance with P2: it is the most recent one if $B R_{i}\left(\alpha_{-i}^{*}\right) \cap\left\{a_{i}^{1}, \ldots, a_{i}^{L}\right\} \neq \emptyset$. For each $i \in N \backslash S$, let $b_{i} \in \lambda_{i}(h, t)$ be the most recent best reply to an arbitrary belief over play in the past $t$ periods. Such a best reply exists by definition of $S$. By $\mathrm{P} 1$ and $\mathrm{P} 2$, the process moves with positive probability from state $h$ to successor $h^{\prime}=\left(b, a^{1}, \ldots, a^{L}\right)$. Now (3) holds by construction: if $i \in N \backslash S$, then $b_{i} \in \lambda_{i}(h, t)$, so $\lambda_{i}(h, t)=\lambda_{i}\left(h^{\prime}, t+1\right)$, and if $i \in S$, then $b_{i} \notin \lambda_{i}(h, t)$, so $\lambda_{i}(h, t) \subset \lambda_{i}(h, t) \cup\left\{b_{i}\right\}=\lambda_{i}\left(h^{\prime}, t+1\right)$.

(b) Fix, for each $i \in N$, a belief $\alpha_{-i} \in \times_{j \in N \backslash\{i\}} \Delta\left(\lambda_{j}(h, t)\right)$ over the play in the past $t$ periods. Since $\times_{i \in N} \lambda_{i}(h, t)$ is a prep set, there is an action $b_{i} \in \lambda_{i}(h, t)$ which is the most recent best reply to this belief. By P1 and P2, the process moves with positive probability from $h$ to $h^{\prime}=\left(b, a^{1}, \ldots, a^{L}\right)$. Since $b_{i} \in \lambda_{i}(h, t)$ for all $i \in N$, it follows that $\lambda_{i}\left(h^{\prime}, t+1\right)=\lambda_{i}(h, t)$, so (4) holds.

Applying Lemma A.1 $T-1$ times, one can construct a sequence $g_{1}, \ldots, g_{T}$ in $H$ with $g_{1}:=h_{0}$ and for all $k=1, \ldots, T-1: P\left(g_{k}, g_{k+1}\right)>0$ and

$$
\times_{i \in N} \lambda_{i}\left(g_{k}, k\right) \subseteq \times_{i \in N} \lambda_{i}\left(g_{k+1}, k+1\right),
$$


with strict inclusion if $\times_{i \in N} \lambda_{i}\left(g_{k}, k\right)$ is not a prep set and equality otherwise. The sequence of product sets $\times_{i \in N} \lambda_{i}\left(g_{k}, k\right)$ in $A$ can increase strictly during at most $\sum_{i \in N}\left|A_{i}\right|-n$ steps: the action space $A$ is a prep set containing $\sum_{i \in N}\left|A_{i}\right|$ actions; $\times_{i \in N} \lambda_{i}\left(g_{1}, 1\right)$ captures $n$ of them, and in each step at least one action is added until a prep set is reached. Hence, the sequence has to reach, after $K \leq \sum_{i \in N}\left|A_{i}\right|-n$ steps, a state $g_{K+1} \in H$ where $\times_{i \in N} \lambda_{i}\left(g_{K+1}, K+1\right)$ is a prep set. ${ }^{6}$ In the final $T-K-1$ steps, we proceed to a state $g_{T}$, where

$$
\times_{i \in N} \lambda_{i}\left(g_{T}, T\right)=\times_{i \in N} \lambda_{i}\left(g_{T-1}, T-1\right)=\cdots=\times_{i \in N} \lambda_{i}\left(g_{K+1}, K+1\right)
$$

remains a prep set. Taking $h_{1}:=g_{T}$ finishes the proof of Step 1 .

\section{A.2 States without blocked actions}

In this section, we show that from a state $h \in H$ such that $\times_{i \in N} \lambda_{i}(h, T)$ is a prep set, the process moves with positive probability within a finite number of steps to a state $h^{\prime} \in H$ where $\times_{i \in N} \lambda_{i}\left(h^{\prime}, T\right) \subseteq \times_{i \in N} \lambda_{i}(h, T)$ is a prep set without blocked actions. This is established in Lemma A.3, using Lemma A.2. Furthermore, in Lemma A.4 we show that when considering a sequence $g_{1}, \ldots, g_{K}$ such that, for all $k=1, \ldots, K$, $\times_{i \in N} \lambda_{i}\left(g_{k}, T\right)$ is a prep set and $\times_{i \in N} \lambda_{i}\left(g_{1}, T\right) \supseteq \cdots \supseteq \times_{i \in N} \lambda_{i}\left(g_{K}, T\right)$, we can assume without loss of generality that none of the states $\left(g_{k}\right)_{k=1, \ldots, K}$ contains a blocked action. We use this result in the lemmata of the following subsections.

Lemma A.2 Let $h \in H$ be such that $\times_{i \in N} \lambda_{i}(h, T)$ is a prep set. For each player $i \in N$, define $\beta_{i}(h) \in \lambda_{i}(h, T)$ as follows:

- if $\tau_{i}(h)$ is blocked, let $\beta_{i}(h) \in \lambda_{i}(h, T)$ be an arbitrary non-blocked action;

- if $\tau_{i}(h)$ is not blocked, let $\beta_{i}(h)=\tau_{i}(h)$.

Set $h^{\prime}=(\beta(h) ; h)$, with $\beta(h)=\left(\beta_{i}(h)\right)_{i \in N}$. Then:

$$
\begin{gathered}
P\left(h, h^{\prime}\right)>0 \\
\times_{i \in N} \lambda_{i}\left(h^{\prime}, T\right) \subseteq \times_{i \in N} \lambda_{i}(h, T) \\
\times_{i \in N} \lambda_{i}\left(h^{\prime}, T\right) \quad \text { is a prep set. }
\end{gathered}
$$

Proof For all $i \in N, \beta_{i}(h) \in \lambda_{i}(h, T)$ is not blocked by definition: there is a belief $\alpha_{-i} \in \times_{j \in N \backslash\{i\}} \Delta\left(\lambda_{j}(h, T)\right)$ against which $\beta_{i}(h)$ is the most recent best reply. By $\mathrm{P} 1$ and P2, (5) holds. Since $\beta_{i}(h) \in \lambda_{i}(h, T)$ for all $i \in N,(6)$ holds. To prove (7), let $i \in N$ and $\alpha_{-i} \in \times_{j \in N \backslash\{i\}} \Delta\left(\lambda_{j}\left(h^{\prime}, T\right)\right)$. To show: $B R_{i}\left(\alpha_{-i}\right) \cap \lambda_{i}\left(h^{\prime}, T\right) \neq \emptyset$. By construction, $\lambda_{i}\left(h^{\prime}, T\right)$ equals either $\lambda_{i}(h, T)$ or, if $\tau_{i}(h)$ was blocked and chosen only once in the most recent $T$ periods of history $h, \lambda_{i}(h, T) \backslash\left\{\tau_{i}(h)\right\}$. Consequently, $\lambda_{i}\left(h^{\prime}, T\right)$ still contains a best reply to every belief over $\times_{j \in N \backslash\{i\}} \Delta\left(\lambda_{j}(h, T)\right)$, in particular to every belief over the subset $\times_{j \in N \backslash\{i\}} \Delta\left(\lambda_{j}\left(h^{\prime}, T\right)\right)$.

6 This motivates the term $M:=\sum_{i \in N}\left|A_{i}\right|-n+1$ in the lower bound on $T$ in (1): reaching a prep set can take $M-1$ steps; recalling the added actions and those in $g_{1}$ can consequently take a memory length $M$. 
Claim (6) means that we weakly decrease the pool of feasible beliefs in going from $h$ to $h^{\prime}=(\beta(h) ; h)$. This implies that if $a_{i}:=\tau_{i}(h)$ was blocked in $h$, but was chosen more than once in the last $T$ rounds of $h$, i.e., if $a_{i} \in \lambda_{i}\left(h^{\prime}, T\right)$, then it remains blocked:

if $a_{i}:=\tau_{i}(h)$ was blocked in $h$ and $a_{i} \in \lambda_{i}\left(h^{\prime}, T\right)$, then it is blocked in $h^{\prime}$.

By definition, blocked actions are not chosen in going from $h$ to $h^{\prime}$. Thus, if an action is blocked in $h$, it is either no longer contained in $\times_{i \in N} \lambda_{i}\left(h^{\prime}, T\right)$, in which case (6) holds with strict inclusion, or it remains blocked in $h^{\prime}$ by (8), but lies further back in players' memory. Hence, repeated application of Lemma A.2 to the sequence $g_{1}, g_{2}, \ldots$ in $H$ with $g_{1}=h$ and $g_{k+1}=\left(\beta\left(g_{k}\right) ; g_{k}\right)$ for all $k \in \mathbb{N}$, yields that a blocked action disappears from memory in at most $T$ steps, in which case the product set of recent actions has become strictly smaller in the weakly decreasing sequence

$$
\times_{i \in N} \lambda_{i}\left(g_{1}, T\right) \supseteq \times_{i \in N} \lambda_{i}\left(g_{2}, T\right) \supseteq \cdots
$$

By (7), the product set remains a prep set. Since there are only finitely many prep sets, it follows that we eventually reach a state $g_{k}$ without blocked actions. This proves:

Lemma A.3 Let $h \in H$ be such that $\times_{i \in N} \lambda_{i}(h, T)$ is a prep set. Either $h$ contains no blocked actions, or the process moves with positive probability in a finite number of steps to a state $h^{\prime} \in H$ where $\times_{i \in N} \lambda_{i}\left(h^{\prime}, T\right) \subset \times_{i \in N} \lambda_{i}(h, T)$ is a prep set and $h^{\prime}$ contains no blocked actions.

The proof of Step 2 uses so-called drag-to-front operations (Sect. A.3) and neighbor switches (Sect. A.4) to establish the following: Given a state $g_{1} \in H$ where $\mathrm{\times}_{i \in N} \lambda_{i}\left(g_{1}, T\right)$ is a prep set, the process moves with positive probability in a finite number of steps through a sequence of states $g_{1}, g_{2}, \ldots, g_{K}$ such that

$$
\begin{gathered}
\forall k=1, \ldots, K: \times_{i \in N} \lambda_{i}\left(g_{k}, T\right) \text { is a prep set, } \\
\times_{i \in N} \lambda_{i}\left(g_{1}, T\right) \supseteq \times_{i \in N} \lambda_{i}\left(g_{2}, T\right) \supseteq \cdots \supseteq \times_{i \in N} \lambda_{i}\left(g_{K}, T\right),
\end{gathered}
$$

and $g_{K}$ has the property that for some minimal prep set $X=\times_{i \in N} X_{i}$ and each $i \in N$ :

$$
\lambda_{i}\left(g_{K},\left|X_{i}\right|\right)=X_{i},
$$

i.e., for each player $i \in N$, the most recent $\left|X_{i}\right|$ actions are exactly those in $i$ 's component of the minimal prep set $X$. If any of the states $g_{k}$ contains a blocked action, apply Lemma A.3 to move to a state $g^{\prime}$ where $\times_{i \in N} \lambda_{i}\left(g^{\prime}, T\right) \subset \times_{i \in N} \lambda_{i}\left(g_{k}, T\right)$ is a prep set and $g^{\prime}$ contains no blocked actions. Then, we can start the repeated use of dragto-front operations and neighbor switches anew from $g^{\prime}$. Since there are only finitely many prep sets and the prep set $\times_{i \in N} \lambda_{i}\left(g^{\prime}, T\right)$ is strictly contained in $\times_{i \in N} \lambda_{i}\left(g_{k}, T\right)$, we eventually reach in a finite number of steps a state from which we can apply drag-to-front operations and neighbor switches without ever encountering a state with a blocked action. Hence: 
Lemma A.4 In a sequence of states $\left(g_{k}\right)_{k=1, \ldots, K}$ satisfying (9) and (10), obtained using drag-to-front operations and neighbor switches, we may assume w.l.o.g. that none of the states contains a blocked action.

\section{A.3 Drag-to-front operations}

Consider a state $h \in H$ containing no blocked actions for which $\times_{i \in N} \lambda_{i}(h, T)$ is a prep set. Then, by definition, for each $i \in N, \beta_{i}(h)=\tau_{i}(h)$, the action player $i$ chose $T$ periods ago in state $h$ (see Lemma A.2). Hence, in the successor $(\beta(h) ; h)=$ $(\tau(h) ; h)$, this action is dragged to the front of player $i$ 's account of recent past play. For easy reference, call the transition from $h$ to $(\beta(h) ; h)=(\tau(h) ; h)$ a drag-to-front operation.

Suppose some player $j \in N$ has an action $a_{j} \in \lambda_{j}(h, T)$ with frequency $f_{j}\left(h, a_{j}\right)=1$. Since $^{7} T \geq 2\left|A_{j}\right|$ by (1), there must be an action $b_{j} \in \lambda_{j}(h, T)$ with frequency $f_{j}\left(h, b_{j}\right) \geq 3$. By Lemma A.4, and using drag-to-front-operations if necessary, we can assume without loss of generality that player $j$ chose $b_{j}$ exactly $T$ periods ago: $\tau_{j}(h)=b_{j}$. For each player $i \in N$, define $\gamma_{i}(h) \in \lambda_{i}(h, T)$ as follows:

$$
\gamma_{i}(h)= \begin{cases}\tau_{i}(h) & \text { if } i \neq j \\ a_{j} & \text { if } i=j\end{cases}
$$

Set $h^{\prime}=(\gamma(h) ; h)$ with $\gamma(h)=\left(\gamma_{i}(h)\right)_{i \in N}$. Recall: (1) $\gamma_{i}(h) \in \lambda_{i}(h, T)$ for all $i \in N,(2) \times_{i \in N} \lambda_{i}(h, T)$ is a prep set, and (3) no actions in $h$ are blocked; so each $\gamma_{i}(h)$ is the most recent best reply to a belief $\alpha_{-i} \in \times_{k \in N \backslash\{i\}} \Delta\left(\lambda_{k}(h, T)\right)$. By P1 and $\mathrm{P} 2, P\left(h, h^{\prime}\right)>0$.

By construction, $\times_{i \in N} \lambda_{i}\left(h^{\prime}, T\right)=\times_{i \in N} \lambda_{i}(h, T)$ remains a prep set. The frequency of the actions of players $i \neq j$ is unaffected: $\forall i \in N \backslash\{j\}, \forall c_{i} \in \lambda_{i}\left(h^{\prime}, T\right)=\lambda_{i}(h, T)$ : $f_{i}\left(h^{\prime}, c_{i}\right)=f_{i}\left(h, c_{i}\right)$. For player $j$ and $c_{j} \in \lambda_{j}\left(h^{\prime}, T\right)=\lambda_{j}(h, T)$ :

$$
f_{j}\left(h^{\prime}, c_{j}\right)= \begin{cases}f_{j}\left(h, c_{j}\right) & \text { if } c_{j} \notin\left\{a_{j}, b_{j}\right\} \\ f_{j}\left(h, a_{j}\right)+1=2 & \text { if } c_{j}=a_{j} \\ f_{i}\left(h, b_{j}\right)-1 \geq 2 & \text { if } c_{j}=b_{j} .\end{cases}
$$

By going from $h$ to $h^{\prime}$, the number of actions with frequency one has strictly decreased, whereas there is no action with frequency larger than or equal to two whose frequency becomes less than two.

Repeating this process, we eventually reach a state where all actions in the history of recent past play have frequency greater than or equal to 2. By Lemma A.3, we may assume that none of its actions is blocked. This proves:

\footnotetext{
7 This motivates the term $2\left|A_{j}\right|$ in the lower bound on $T$. Moreover, if the memory length is below this bound, neighbor switches as defined in Sect. A.4 may cause actions from prep sets to disappear from a player's recollection.
} 
Lemma A.5 Let $h \in H$ be such that $\times_{i \in N} \lambda_{i}(h, T)$ is a prep set. Then the process moves with positive probability in a finite number of steps to a state $h^{\prime} \in H$ with $\times_{i \in N} \lambda_{i}\left(h^{\prime}, T\right) \subseteq \times_{i \in N} \lambda_{i}(h, T)$ such that

[C1] $\times_{i \in N} \lambda_{i}\left(h^{\prime}, T\right)$ is a prep set,

[C2] all actions have frequency at least $2: \forall i \in N, \forall a_{i} \in \lambda_{i}\left(h^{\prime}, T\right): f_{i}\left(h^{\prime}, a_{i}\right) \geq 2$,

[C3] $h^{\prime}$ contains no blocked actions.

\section{A.4 Neighbor switches}

Repeatedly applying drag-to-front operations starting in a state $h \in H$ where no actions are blocked and $\times_{i \in N} \lambda_{i}(h, T)$ is a prep set, we get a sequence of states $g_{0}, g_{1}, \ldots \in H$ with $g_{0}:=h$ such that for all players $i \in N$ and all $t \in \mathbb{N}: \ell_{i}\left(g_{t}\right)=\tau_{i}\left(g_{t-1}\right)$, i.e., we get a periodic repetition of each player's actions.

Instead, it is possible that some player $i$ chooses his actions in such a way that the process moves to a state in which the order in which player $i$ plays two neighboring actions-say those chosen $t$ and $t+1$ periods ago in state $h$-is changed, while the others continue to play actions in their given order. For instance, the process may move from Fig. 3a to e, where player $i$ 's order of actions $b$ and $c$, chosen 2 and 3 periods ago in Fig. 3a, respectively, is reversed while the order of actions of players $j \neq i$ is unchanged. In Fig. 3, the length of recent past play $T$ is 4; actions chosen during the most recent four periods are contained in the boxed part of the table; actions outside the boxes have disappeared from recent past play. For instance, in Fig. 3c, player $i$ chose $c$ five periods ago, $d$ six periods ago. Since $T=4$, these actions are no longer part of recent past play.

The idea is simple: ${ }^{8}$ use drag-to-front operations until the actions to be switched are those chosen $T-1$ and $T$ periods ago (the transition from Fig. 3a to 3b); in the next two periods, let players $j \neq i$ continue with drag-to-front operations, while player $i$ chooses the actions that are to be switched in reverse order (in going from Fig. $3 \mathrm{~b}$ to $3 \mathrm{c}, i$ chooses $b$ instead of $c$, in going from the Fig. 3c to 3d, $i$ chooses $c$ instead of $b$ ). Finally, use drag-to-front operations until the switched actions are again at coordinates $t$ and $t+1$ in the recent past play (the transition from Fig. 3d to $3 \mathrm{e}$ ). Formally:

Lemma A.6 Let $h \in H$ satisfy [C1] to [C3]. Let $i \in N, t \in\{1, \ldots, T-1\}$. Assuming w.l.o.g. (Lemma A.4) that we encounter no blocked actions, the process moves with positive probability in T steps to a state $h^{\prime} \in H$ satisfying [C1] to [C3] and in which $a_{j}\left(h^{\prime}, k\right)=a_{j}(h, k)$ if $j=i$ and $k \notin\{t, t+1\}$, or if $j \neq i$, whereas $a_{i}\left(h^{\prime}, t\right)=$ $a_{i}(h, t+1)$ and $a_{i}\left(h^{\prime}, t+1\right)=a_{i}(h, t)$.

Proof For notational convenience, let $a_{i}$ and $b_{i}$ be the actions player $i$ chose $t+1$ and $t$ periods ago in $h$, respectively. Performing $T-t-1$ drag-to-front operations, we reach a state $g_{1}$ satisfying [C1] to [C3] in which $a_{i}$ is the action $i$ chose $T$ periods ago and $b_{i}$ the action he chose $T-1$ periods ago.

Construct a successor $g_{2}$ of $g_{1}$ as follows: for each $j \in N \backslash\{i\}$, set $s_{j}^{1}=\tau_{j}\left(g_{1}\right)$ and set $s_{i}^{1}=b_{i}$. Define $g_{2}=\left(s^{1} ; g_{1}\right)$, where $s^{1}=\left(s_{j}^{1}\right)_{j \in N}$.

\footnotetext{
${ }^{8}$ Figure 3 is for illustration only; we assume that all steps we describe there are feasible.
} 
Fig. 3 Switch $i$ 's actions $b$ and $c$, keeping those of players $j \neq i$ in the same order

\begin{tabular}{|c|c|c|c|c|}
\hline player $i:$ & $a$ & $b$ & $c$ & $d$ \\
\hline player $j:$ & $\alpha$ & $\beta$ & $\gamma$ & $\delta$ \\
\hline
\end{tabular}

b

\begin{tabular}{|c|c|c|c|c|}
\hline player $i$ : & $d$ & $a$ & $b$ & $c$ \\
\hline player $j$ : & $\delta$ & $\alpha$ & $\beta$ & $\gamma$ \\
\hline
\end{tabular}

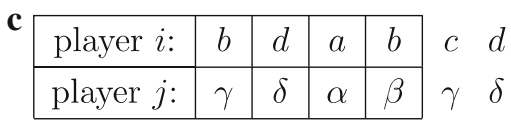

\begin{tabular}{|c|c|c|c|c|ccc|}
\hline player $i:$ & $c$ & $b$ & $d$ & $a$ & $b$ & $c$ & $d$ \\
\cline { 1 - 5 } player $j:$ & $\beta$ & $\gamma$ & $\delta$ & $\alpha$ & $\beta$ & $\gamma$ & $\delta$ \\
\hline
\end{tabular}

\begin{tabular}{|c|c|c|c|c|cccc|}
\hline player $i:$ & $a$ & $c$ & $b$ & $d$ & $a$ & $b$ & $c$ & $d$ \\
\cline { 1 - 5 } & player $j:$ & $\alpha$ & $\beta$ & $\gamma$ & $\delta$ & $\beta$ & $\gamma$ & $\delta$ \\
\hline
\end{tabular}

Construct a successor $g_{3}$ of $g_{2}$ as follows: for each $j \in N \backslash\{i\}$, set $s_{j}^{2}=\tau_{j}\left(g_{2}\right)$ and set $s_{i}^{2}=a_{i}$. Define $g_{3}=\left(s^{2} ; g_{2}\right)$, where $s^{2}=\left(s_{j}^{2}\right)_{j \in N}$.

For players $j \neq i$, these two steps involve simple drag-to-front operations. For player $i$ it involves reversing the order: in going from $g_{1}$ to $g_{2}, i$ chooses $b_{i}$, in going from $g_{2}$ to $g_{3}, i$ chooses $a_{i}$, rather than playing first $a_{i}$, then $b_{i}$.

As $\times_{i \in N} \lambda_{i}\left(g_{1}, T\right)$ is a prep set and no actions are blocked in $g_{1}$, it follows from $\mathrm{P} 1$ and P2 that $P\left(g_{1}, g_{2}\right)>0$. Moreover, as all actions in $h$ have frequency at least 2 , we have that $\lambda_{i}\left(g_{1}, T\right)=\lambda_{i}\left(g_{2}, T\right)$ for all $i \in N$. Hence, also $\times_{i \in N} \lambda_{i}\left(g_{2}, T\right)$ is a prep set. By Lemma A.4 we may assume that $g_{2}$ contains no blocked actions. Hence, also $P\left(g_{2}, g_{3}\right)>0$. Moreover, it is easy to see that frequencies in $g_{3}$ are identical to frequencies in $g_{1}$, i.e., at least equal to 2 . We can thus conclude that also $g_{3}$ satisfies [C1] to [C3].

In $g_{3}$, the two actions that are played most recently are $a_{i}$ and $b_{i}$, respectively. Thus, performing $t-1$ drag-to-front operations leads to the desired state $h^{\prime}$.

\section{A.5 Proof of steps $2-4$}

Step 2 Let $h_{1} \in H$ be such that $\times_{i \in N} \lambda_{i}\left(h_{1}, T\right)$ is a prep set. The process moves with positive probability in a finite number of steps to a state $h_{2} \in H$ where $\times_{i \in N} \lambda_{i}\left(h_{2}, T\right)$ is a minimal prep set.

Proof By Lemma A.5, the process moves with positive probability in a finite number of steps from $h_{1}$ to a state $g \in H$ satisfying [C1] to [C3]. Let $X=\times_{i \in N} X_{i} \subseteq$ $\times_{i \in N} \lambda_{i}(g, T)$ be a minimal prep set. Assuming w.l.o.g. (Lemma A.4) that from $g$ onward we do not encounter blocked actions, Lemma A.6 allows us to perform neighbor switches. Every permutation of a finite set can be obtained by a chain of neighbor switches; thus, repeated application of Lemma A.6 yields that the process moves in a 
finite number of steps to a state $g_{0} \in H$ with the property that for each player $i \in N$, $\lambda_{i}\left(g_{0},\left|X_{i}\right|\right)=X_{i}$, i.e., for each player $i \in N$, the most recent $\left|X_{i}\right|$ actions in $g_{0}$ are exactly those in $i$ 's component of the minimal prep set $X$.

For each $k \in \mathbb{N}$, let $g_{k}:=\left(\left(a_{i}\left(g_{k-1},\left|X_{i}\right|\right)\right)_{i \in N} ; g_{k-1}\right) \in H$, i.e., $g_{k}$ is the successor of $g_{k-1}$ obtained by letting each player $i \in N$ play the action he chose $\left|X_{i}\right|$ periods ago in $g_{k-1}$. Recalling that $X$ is a minimal prep set, a simple inductive proof establishes that for all $k \in \mathbb{N}$ it holds that $P\left(g_{k-1}, g_{k}\right)>0$ and for all players $i \in N$ we have

$$
\lambda_{i}\left(g_{k}, \min \left\{\left|X_{i}\right|+k, T\right\}\right)=X_{i} .
$$

Set $k=T$ to find that $\times_{i \in N} \lambda_{i}\left(g_{T}, T\right)=X$. Taking $h_{2}:=g_{T}$ finishes the proof of Step 2.

Step 3 Let $h_{2} \in H$ be such that $X=\times_{i \in N} \lambda_{i}\left(h_{2}, T\right)$ is a minimal prep set. After reaching $h_{2}$, all action profiles that are played with positive probability lie in $X$ :

$$
\forall k \in \mathbb{N}, \forall h \in H: P^{k}\left(h_{2}, h\right)>0 \Rightarrow \ell(h) \in X \text {. }
$$

Proof By P1 and P2, players always base beliefs on the actions played in the last $T$ periods and choose the most recent best reply to such beliefs. In $h_{2}$, their account of recent play $\times_{i \in N} \lambda_{i}\left(h_{2}, T\right)$ equals the minimal prep set $X$, which by definition contains at least one best reply to whatever belief a player may have about other players' choices from $X$. Hence, by induction, the actions from minimal prep set $X$ will always be fresher in players' recollection of past play than actions outside $X$, i.e., beliefs and best replies to these beliefs will, by P1 and P2, always have support in $X$. Formally, for all $k \in \mathbb{N}$ and $h \in H$ :

$$
\text { if } P^{k}\left(h_{2}, h\right)>0 \text {, then } \times_{i \in N} \lambda_{i}(h, T+k)=X,
$$

and hence

$$
\times_{i \in N} \lambda_{i}(h, T) \subseteq X
$$

In particular, this means $\ell(h) \in X$, i.e., (11) holds.

Step 4 For every state $h_{0} \in H$, the process eventually reaches a state $h_{2} \in H$ satisfying the conditions in Step 2, i.e., where according to Step 3 play settles down in a minimal prep set.

Proof Call two states $h=\left(a^{1}, \ldots, a^{L}\right)$ and $g=\left(b^{1}, \ldots, b^{K}\right)$ in $H$ equivalent, denoted $h \sim g$, if they have the same account of recent past play and the same order in which each player $i$ 's actions are encountered:

$$
h \sim g \Leftrightarrow\left\{\begin{array}{l}
\left(a^{1}, \ldots, a^{T}\right)=\left(b^{1}, \ldots, b^{T}\right), \\
o_{i, h}=o_{i, g} \text { for all } i \in N .
\end{array}\right.
$$

Notice that $\sim$ is an equivalence relation on $H$; for each $h \in H$, let $[h]=\left\{h^{\prime} \in H: h \sim\right.$ $h^{\prime}$ \} be the equivalence class containing $h$. Recall from Sect. 3 that in each state $h \in H$, 
if the sequence of action profiles from the past $T$ periods is $\left(a^{1}, \ldots, a^{T}\right) \in A^{T}$, then, firstly, player $i$ 's beliefs $\alpha_{-i}$ are drawn from a probability distribution $\mathbb{P}_{\left(i,\left(a^{1}, \ldots, a^{T}\right)\right)}$ and, secondly, his response is (whenever possible) the most recent best reply to this belief or (otherwise) drawn from a probability distribution $\mathbb{P}_{\alpha_{-i}}$ over his best replies. Thus, player $i$ 's choice behavior is the same in two equivalent states. Since there are only finitely many elements in $A^{T}$ and $N$, it follows that the set of positive transition probabilities $\left\{P\left(h, h^{\prime}\right) \mid h, h^{\prime} \in H, P\left(h, h^{\prime}\right)>0\right\}$ is a finite set. Let $\varepsilon>0$ be its minimum.

By Steps 1 to 3, it is possible, from any history $h_{0} \in H$, to reach a state $h_{2} \in H$ in an absorbing set where play settles down in a minimal prep set in a finite number of steps, say $k\left(h_{0}\right) \in \mathbb{N}$. By definition of equivalence, $k(h)=k\left(h_{0}\right)$ for all $h \in\left[h_{0}\right]$ : the set $\left\{k\left(h_{0}\right) \mid h_{0} \in H\right\}$ is finite. Let $\kappa \in \mathbb{N}$ be its minimum.

By definition of $\varepsilon$ and $\kappa$, the probability of entering an absorbing set where play settles down in a minimal prep set in at most $\kappa$ steps is at least $\varepsilon^{\kappa}$ from any state. Hence, the probability of not reaching an absorbing set in $\kappa$ steps is at most $1-\varepsilon^{\kappa}$, which is less than 1 . So the probability of not reaching an absorbing set in $k \kappa$ steps is less than or equal to $\left(1-\varepsilon^{\kappa}\right)^{k}$, which goes to zero as $k$ goes to infinity.

\section{Appendix B Proof of proposition 5.1}

Proof (The inclusion $\mathscr{P} \subseteq \widetilde{\mathscr{P}}$ ): Let $P \in \mathscr{P}$. The probability $R_{i, h}\left(a_{i}\right)$ that player $i \in N$ in state $h=\left(a^{1}, \ldots, a^{L}\right) \in H$ chooses action $a_{i} \in A_{i}$ equals the probability of drawing a belief $\alpha_{-i}$ from $\mathbb{P}_{\left(i,\left(a^{1}, \ldots, a^{T}\right)\right)}$ to which:

(i) $a_{i}$ is the most recent best reply, or, alternatively,

(ii) no best reply was played before, but response $a_{i}$ is drawn from $\mathbb{P}_{\alpha_{-i}}$.

Hence, there are functions $R=\left(R_{i, h}\right)_{i \in N, h \in H}$ such that $P=P_{R}$. Conditions $(\alpha)$ and $(\beta)$ follow trivially from $\mathrm{P} 1$ and $\mathrm{P} 2$ in the definition of $\mathscr{P}$. Conclude that $P \in \widetilde{\mathscr{P}}$. (Convergence:) The proof of Theorem 4.1 in Appendix A applies with minor changes to $P_{R}$ as well:

- condition $(\alpha)$ guarantees that Steps 1 and 2 hold without change,

- condition $(\beta)$ guarantees that Step 3 holds without change,

- by (2), there are only finitely many different functions in $R=\left(R_{i, h}\right)_{i \in N, h \in H}$, so the equivalence relation in Step 4 is well defined and there are again finitely many equivalence classes; hence, also Step 4 holds.

\section{References}

Basu K, Weibull JW (1991) Strategy subsets closed under rational behavior. Econ Lett 36:141-146

Crawford VP, Haller H (1990) Learning how to cooperate: optimal play in repeated coordination games. Econometrica 58:571-595

Ellison G, Fudenberg D (1993) Rules of thumb for social learning. J Polit Econ 101:612-643

Fudenberg D, Levine DK (1998) The theory of learning in games. MIT Press, Cambridge

Grossman SJ, Kihlstrom RE, Mirman LJ (1977) A Bayesian approach to the production of information and learning by doing. Rev Econ Stud 44:533-547

Hurkens S (1995) Learning by forgetful players. Games Econ Behav 11:304-329 
Joosten R, Peters H, Thuijsman F (1995) Unlearning by not doing: repeated games with vanishing actions. Games Econ Behav 9:1-7

Kalai E, Samet D (1984) Persistent equilibria in strategic games. Int J Game Theory 14:41-50

Kets W, Voorneveld M (2007) Congestion, equilibrium and learning: the minority game. CentER Discussion Paper 2007-61

Madrian BC, Shea DF (2001) The power of suggestion: inertia in 401(k) participation and savings behavior. Quart J Econ 116:1149-1187

Miller N, Campbell DT (1959) Recency and primacy in persuasion as a function of the timing of speeches and measurements. J Abnormal Soc Psychol 59:1-9

Schelling T (1960) The strategy of conflict. Harvard University Press, Cambridge

Tercieux O, Voorneveld M (2005) The cutting power of preparation. SSE/EFI Working paper series in Economics and Finance, No. 583, Stockholm School of Economics

Tversky A, Kahneman D (1982) Judgment under uncertainty: heuristics and biases. In: Kahneman D, Slovic P, Tversky A (eds) Judgment under uncertainty: heuristics and biases. Cambridge University Press, Cambridge, pp 3-20

Vega-Redondo F (1993) Simple and inertial behavior: an optimizing decision model with imprecise perceptions. Econ Theory 3:87-98

Voorneveld M (2004) Preparation. Games Econ Behav 48:403-414

Voorneveld M (2005) Persistent retracts and preparation. Games Econ Behav 51:228-232

Voorneveld M, Kets W, Norde H (2005) An axiomatization of minimal curb sets. Int J Game Theory 33:479-490

Young HP (1998) Individual strategy and social structure. Princeton University Press, Princeton 